\title{
A TWO LEVEL DECENTRALIZED DISTRIBUTION SYSTEM WITH COMPOUND RENEWAL DEMAND
}

\author{
EMŐKE BÁZSA* AND PETER DEN ISEGER ${ }^{\dagger}$ \\ Econometric Institute Report EI 2002-45
}

\begin{abstract}
In this paper we consider a two level decentralized distribution system, consisting of one warehouse and $N$ retailers. The warehouse and each retailer follows each his own(s,nQ) order policy. We extended the models as known in the literature to compound renewal demand.
\end{abstract}

\section{INTRODUCTION}

Within supply chain management the most commonly encountered problem is the modeling and optimization of the so called multi echelon production/distribution systems. These systems are also called networks. The simplest structure is a series system, where the output of each production or stocking point represents the input of the successive stage, hence each stage supplies the next one (cf. [5]). There is only one finished product at the end of the system, where demand occurs. To follow with we should mention the assembly system: here is also one finished product, although there may be several raw materials, all supplied exogenously. These are assembled into components, then in further stages assembled further finally into the end product. A distribution system if represented on a diagram looks like a reversed assembly system (cf. [5]). Further there are tree systems, which allow features of both assembly and distribution systems and general systems which allow more complex features. In this paper we consider a distribution system, restricting the analysis to only two stages, which is very common to do in the literature.

\footnotetext{
Date: December 16, 2002.

*Erasmus University Rotterdam, Econometric Institute, P.O. Box 1738, 3000 DR Rotterdam, The Netherlands, e-mail: bazsa@few.eur.nl.

${ }^{\dagger}$ Erasmus University Rotterdam, Econometric Institute, P.O. Box 1738, 3000 DR Rotterdam, The Netherlands, e-mail: iseger@few.eur.nl.
} 
Information and control can be centralized or decentralized (localized). In the latter case each retailer sees only its own demands and the warehouse sees only the incoming order streams. The warehouse applies a first-come-first-served rule, and all the locations apply local policies. On the other hand, with a centralized control, the warehouse already accounts for demands when they occur at the retailers, thus it requires fully centralized information. Hence, the essential difference between centralized and decentralized control systems is, that the latter relies on the history rather then the current status of the system to make crucial decisions (cf. [5]). The centralized control system works well for high-volume goods, while the decentralized approach is better for low-volume items. In the first case the retailers often compete for shipments, while for low volume items this is rarely the case, and most of the time the shipments are more or less in the same size category (thus a first-come-first-served rule makes sense). The system analyzed in this paper is a decentralized two-level distribution system.

The classic model of a multi-level distribution system is METRIC, developed by Sherbrooke (cf. [3]). He also assumes only two stages, demand at the retailers Poisson, all locations using a base stock - $(S-1, S)$ - policy. Variations on the METRIC models are also developed, although they could only consider base stock policies. Deuermeyer and Schwarz (cf. [2]) published in 1981 one of the first articles considering decentralized distribution systems with non base stock policies. They consider a one warehouse multiple identical retailers system, where external demand is Poisson (unit), retailers apply $(\mathrm{s}, \mathrm{Q})$ policies. One of the major limitations of their model is that it assumes 'no delay due to warehouse out-of-stock conditions'.

\section{THE MODEL}

Consider a two level distribution system, consisting of one warehouse and $N$ retailers. The warehouse and retailers follow $(s, n Q)$ control policies, that is, the warehouse applies a policy with parameters $\left(s_{0}, Q_{0}\right)$, while at retailer $i$ the policy parameters are $\left(s_{i}, Q_{i}\right), i=1, \ldots, N$. All the processes and characteristics describing or related to the retailers will be indexed with $i, i=1, \ldots, N$, while for the warehouse we use index 0 . Let $\left\{\mathbf{I P}_{i}(t): t \geq 0\right\}, i=0, \ldots, N$ denote the inventory position processes. 
Lemma 2.1. Let $\mathbf{U}$ be uniform distributed on $[0,1]$ and let $\mathbf{a}$ be a positive random variable.. Then $(\mathbf{U}+\mathbf{a}) \bmod 1$ is uniform distributed on $[0,1]$. Moreover, $(\mathbf{U}+\mathbf{a}) \bmod 1$ is independent of $\mathbf{a}$.

Proof: It is sufficient to prove that for all $\alpha \geq 0,(\mathbf{U}+\alpha) \bmod 1$ is uniform distributed on $[0,1]$. This can be verified by a simple calculation.

This result has an important consequence for the IP under an $(s, n Q)$ control policy.

Corollary 2.2. Suppose $\mathbf{I P}(0)$ is uniform distributed on $[s, s+Q)$. For a $(s, n Q)$ control policies and all fixed $t \geq 0, \mathbf{I P}(t)$ is independent of the demand process. Moreover, for all fixed $t \geq 0, \mathbf{I P}(t)$ is uniform distributed on $(s, s+Q]$.

Proof: It is well-known that $\mathbf{I P}(t)-s=(\mathbf{I P}(0)-s+\mathbf{D}(t)) \bmod Q$. Hence the result follows from lemma 2.1.

Assumption 2.3. For all the retailers and the warehouse $\mathbf{I P}_{i}(0)$ is uniform distributed on $\left[s_{i}, s_{i}+Q_{i}\right)$.

Demand is denoted by $\mathbf{D}_{i}$. Identifying the replenishment moments $\tau_{i}=\left\{\tau_{i}^{n}: n \in \mathbb{N}\right\}$ Let us denote the stochastic counting process associated with the sequence $\tau_{i}$ by $\mathbf{N}_{i}(t)$, $i=1, \ldots, N$. Introduce the stochastic replenishment process by

$$
\mathbf{R}_{i}(t):=\sum_{n=1}^{\mathbf{N}_{i}(t)} \mathbf{R}_{i}^{n},
$$

where the $\mathbf{R}_{i}^{n}$ denote the sizes of the replenishment orders. Let further, $L_{i}, i=0, \ldots, N$ stands for the leadtime of a replenishment order. Demand at the warehouse is the superposition of the replenishment processes $\left(\tau_{i}, \mathbf{R}_{i}\right)$ of the retailers, that is,

$$
\mathbf{D}_{0}(0, t]=\sum_{i=1}^{N} \mathbf{R}_{i}(t) .
$$

Theorem 2.4. The replenishment process $\mathbf{R}_{i}$ is distributed as

$$
\begin{aligned}
& \mathbf{R}\left(t_{0}, t_{1}\right]=\left\lfloor\mathbf{D}\left(t_{0}, t_{1}\right]-\left((s+Q)-\mathbf{I P}\left(t_{0}\right)\right)\right\rfloor_{Q} \\
& \mathbf{R}\left(t_{0}, t_{1}\right]=\left\lfloor\mathbf{D}\left(t_{0}, t_{1}\right]+\left(\mathbf{I P}\left(t_{1}\right)-s\right)\right\rfloor_{Q},
\end{aligned}
$$

where $\lfloor x\rfloor_{Q}$ denotes $Q\lfloor x / Q\rfloor$. Moreover, $\mathbf{R}$ is distributed as

$$
\mathbf{R}\left(t_{0}, t_{1}\right]=\left\lfloor\mathbf{D}\left(t_{0}, t_{1}\right]+Q \mathbf{U}\right\rfloor_{Q},
$$


with $\mathbf{U}$ and $\mathbf{D}$ are independent.

Proof: It is easily seen that

$$
\mathbf{R}\left(t_{0}, t_{1}\right]-\left(\mathbf{I P}\left(t_{1}\right)-s\right)=\mathbf{D}\left(t_{0}, t_{1}\right]-\left(\mathbf{I P}\left(t_{0}\right)-s\right)
$$

Since $\mathbf{R}_{i}$ is always a multiple of $Q$ and $0 \leq \mathbf{I P}-s<Q$ we obtain that

$$
\begin{aligned}
& \mathbf{R}\left(t_{0}, t_{1}\right]=\left\lfloor\mathbf{D}\left(t_{0}, t_{1}\right]+\left((s+Q)-\mathbf{I P}\left(t_{0}\right)\right)\right\rfloor_{Q} \\
& \mathbf{R}\left(t_{0}, t_{1}\right]=\left\lfloor\mathbf{D}\left(t_{0}, t_{1}\right]+\left(\mathbf{I P}\left(t_{1}\right)-s\right)\right\rfloor_{Q} .
\end{aligned}
$$

The remainder part follows now from corollary 2.2 .

Remark 2.5. Remark that until now we didn't make any assumption about the demand processes $\mathbf{D}_{i}$.

Let us proceed with the analysis of the warehouse.

\section{The Warehouse}

In order to compute the long run average cost of the warehouse, related to the netstock process, we make use of the flow conservation law:

$$
\mathbf{I N}_{0}\left(t+L_{0}\right)=\mathbf{I P}_{0}(t)-\mathbf{D}_{0}\left(t, t+L_{0}\right]
$$

In the spirit of Bázsa and den Iseger [1], to be able to use an efficient procedure, we need to find out whether the inventory position process and the leadtime demand are asymptotically independent. But this follows immediately from corollary 2.2. We also know that for all $t \geq 0$, $\mathbf{I P}_{0}(t)$ is uniform distributed on $\left[s_{0}, s_{0}+Q_{0}\right)$. It remains to analyze the (Cesaro) limiting distribution of $\mathbf{D}_{0}\left(t, t+L_{0}\right]$. From theorem 2.4 we obtain that

$$
\mathbf{R}_{i}\left(t, t+L_{0}\right]=\left\lfloor\left(\mathbf{D}_{i}\left(t, t+L_{0}\right]+Q_{i} \mathbf{U}\right)\right\rfloor_{Q_{i}},
$$

Assumption 3.1. Demand, denoted by $\mathbf{D}_{i}$, at each retailer is described by a compound renewal process with i.i.d. individual demands $\left\{\mathbf{Y}_{i}^{n}: n \in \mathbb{N}\right\}, i=1, \ldots, N$. The inter-arrival time distributions are spread-out (cf. Sigman [4]).

$\mathbf{D}_{i}\left(t, t+L_{0}\right]$ is distributed as $\mathbf{D}_{i}\left[0, L_{0}-A_{i}(t)\right]$, where $A_{i}$ the forward recurrence time residual life processes $\mathbf{A}_{i}(t):=\mathbf{t}_{\mathbf{N}_{i}(t)+1}-t$. Since the inter-arrival time distributions are spread-out 
the stochastic process $\mathbf{A}_{i}(t)$ has a limiting distribution (cf. Proposition 5.1 of Sigman cf. [4]). This implies that $\mathbf{R}_{i}\left(t, t+L_{0}\right]$ and $\mathbf{D}_{0}\left(t, t+L_{0}\right]$ are asymptotic distributed as

$$
R_{i}^{L_{0}}:=\left\lfloor\left(\mathbf{D}_{i}\left[0, L_{0}-A_{i}^{\infty}\right]+Q_{i} \mathbf{U}\right)\right\rfloor_{Q_{i}} \text { and } \mathbf{D}_{0}^{L_{0}}=\sum_{i=1}^{N} R_{i}^{L_{0}}
$$

where $A_{i}^{\infty}$ is distributed with the limiting distribution of $\mathbf{A}_{i}(t)$. Hence, the average cost of the warehouse is given by the expression

$$
\mathbb{E} f\left(s_{0}+Q_{0} \mathbf{U}-\mathbf{D}_{0}^{L_{0}}\right) .
$$

It remains now to analyze the behaviour of the relevant stochastic processes at the retailers.

\section{The Retailers}

The flow conservation law for the retailers is given by

$$
\mathbf{I N}_{i}\left(t+L_{i}+\mathbf{W}(t)\right)=\mathbf{I P}_{i}(t)-\mathbf{D}_{i}\left(t, t+L_{i}+\mathbf{W}(t)\right]
$$

where $\mathbf{W}(t)$ is the additional remaining waiting time at time $t$ incurred by the event when the warehouse is out of stock. By this definition of the waiting time it is clear that the flow conservation law remains valid. In general the difficulty is to determine the distribution of the remaining waiting time $\mathbf{W}(t)$.

Let us first attempt to determine the distribution of the remaining waiting time at $t, \mathbf{W}(t)$, since, intuitively, that will also reveal the precise dependency structure. The state space of $\mathbf{W}(t)$ is $\left[0, L^{0}\right]$, and its distribution can be characterized by the following relation:

$$
\begin{aligned}
\mathbb{P}\{\mathbf{W}(t) \leq w\} & =\mathbb{P}\left\{\mathbf{I N}_{0}(t+w)+\mathbf{D}_{0}(t, t+w]>0\right\} \\
& =\mathbb{P}\left\{\mathbf{I P}_{0}\left(t+w-L_{0}\right)-\mathbf{D}_{0}\left(t+w-L_{0}, t\right]>0\right\}
\end{aligned}
$$

Intuitively, relation (4.2) expresses the fact that if the remaining waiting time is $w$, then the incoming order at the warehouse at time $t+w$ raises the net inventory of the warehouse $\mathbf{I N}_{0}$ to a positive level, if no demand is coming in at the warehouse during the time $[t, t+w]$. Hence, the distribution of the remaining waiting time only depends on the retailer in question indirectly, through the demand $\mathbf{D}_{0}(t, t+w]$. In relation (4.3) the flow conservation law is used for $\mathbf{I N}_{0}(t+w)$.

The demand, as defined earlier, $\mathbf{D}_{0}\left(t+w-L_{0}, t\right]=\sum_{k=1}^{N} \mathbf{R}_{i}\left(t+w-L_{0}, t\right]$. All the $\mathbf{R}_{k}, k=1, \ldots N$ are independent, and we can determine the superposition $\mathbf{D}_{0}$ as before. 
However, we have to take care for the dependency of $\mathbf{W}(t)$ and $\left\{\mathbf{I P}_{i}(t), \mathbf{D}_{i}(t, t+w]\right\}$. We obtain from theorem 2.4 that

$$
\mathbf{R}_{i}\left(t+w-L_{0}, t\right]=\left\lfloor\mathbf{D}_{i}\left(t+w-L_{0}, t\right]+\mathbf{I P}_{i}(t)-s_{i}\right\rfloor_{Q_{i}}
$$

with $\mathbf{D}_{i}\left(t+w-L_{0}, t\right]$ and $\mathbf{I} \mathbf{P}_{i}(t)-s_{i}$ are independent. This implies that given the forward recurrence time residual life processes $\mathbf{A}_{i}(t):=\mathbf{t}_{\mathbf{N}_{i}(t)+1}-t, \mathbf{W}(t)$ is independent of $\mathbf{D}_{i}(t, t+w]$. Hence, the average cost of retailer $i$ is given by the expression

$$
\mathbb{E}_{\mathbf{U}, A_{i}^{\infty}}\left\{\mathbb{E}\left\{f\left(\mathbf{I P}_{i}-\mathbf{D}_{i}\left[0, L_{i}+\mathbf{W}-A_{i}^{\infty}\right]\right)\right\} \mid A_{i}^{\infty}, \mathbf{I P}_{i}-s_{i}=Q_{i} \mathbf{U}\right\}
$$

Observation 4.1. The waiting time $\mathbf{W}$ with distribution given by (4.3), is not only important for the calculation of long run average costs, but it yields service measure, frequently used in practice: the probability that a customer has to wait more than certain amount of time $T>0$.

Remark 4.2. The analysis of the two level distribution system described above remains valid also in the case when the system starts in an arbitrary state, not in equilibrium. In this case we need the additional assumption of a finite expected delay-cycle cost.

\section{REFERENCES}

1. E.M. Bázsa and P.W. den Iseger, Single item inventory models: A time and event averages approach, Discussion Paper Series 101/4, Tinbergen Institute, Rotterdam, 2001, Submitted to Operations Research.

2. B. Deuermeyer and L. Schwarz, A model for analysis of system level in warehouse- retailer distribution systems: The identical retailer case, TIMS studies in Management Sciences 16 (1981), 163-193.

3. C.C. Sherbrooke, METRIC: A multi-echelon technique for recoverable item control, Operations Research 16 (1968), 122-141.

4. K. Sigman, One-dependent regenerative processes and queues in continuous time, Mathematics of Operations Research 15 (1990), no. 1, 175-189.

5. P.H. Zipkin, Foundations of inventory management, Operations and Decision Sciences, McGraw-Hill, Higher Education Div., Boston, 2000. 